\title{
Immunosuppression mediated by enzymes that degrade tryptophan
}

\author{
B. Van den Eynde \\ Ludwig Institute for Cancer Research, Brussels Branch, Brussels, Belgium \\ Université catholique de Louvain, de Duve Institute, Brussels,Belgium
}

Both central and peripheral mechanisms ensure the tight regulation of the immune system that is needed to ensure efficient protection against pathogens while avoiding autoimmunity. Peripheral immune tolerance mechanisms include negative feedback pathways controlled by immune checkpoints, whose function is to shut down established immune responses and prevent excessive immune activation. These immune checkpoints normally act when the pathogen has been cleared. The immune system also attacks tumors, but the kinetics is often slower, so that immune checkpoints get activated before complete clearance of the tumor. In recent years, a better understanding of immune checkpoints has allowed the design of new cancer therapies able to sustain immune responses by blocking immune checkpoints such as PD-1 and CTLA4. Those new therapies have shown unprecedented clinical activity in a subset of patients with metastatic cancers. However, other immune checkpoints likely limit the efficacy of cancer immunotherapy in other patients. Ongoing research aims at characterizing these additional mechanisms and devising therapeutic strategies to overcome them. One of them is based on metabolic changes in the tumor microenvironment: many tumors aberrantly express enzymes able to degrade tryptophan into kynurenine. These are indoleamine-dioxygenase (IDO) and, to a lesser extent, tryptophan dioxygenase (TDO). These metabolic changes are profoundly immunosuppressive, through the combined action of tryptophan depletion and kynurenine production. Inhibitors of these enzymes increase the efficacy of cancer immunotherapy in preclinical models and are currently in clinical development. The exact mechanisms responsible for IDO/TDO-induced immunosuppression remain unclear. Different pathways have been proposed, including a GCN2-mediated integrated stress response or a deactivation of mTOR, both triggered by tryptophan shortage, and the induction of an AHR response by kynurenine or its derivatives. 